\title{
Know Your Response: Pilot Mapping of HIV Prevention Responses by Non-governmental Organizations in Southern Province, Zambia
}

\author{
Mbozi, Parkie ${ }^{1, *}$, Anastasia J. Gage ${ }^{2}$, Munukayumbwa Munyima ${ }^{1}$, Mubiana Macwang'wi ${ }^{1}$ \\ ${ }^{1}$ Institute of Economic and Social Research, University of Zambia, Lusaka, Zambia \\ ${ }^{2}$ Department of Global Community Health and Behavioral Sciences, Tulane University, New Orleans, Louisiana, United States
}

Copyright $\mathrm{O} 2018$ by authors, all rights reserved. Authors agree that this article remains permanently open access under the terms of the Creative Commons Attribution License 4.0 International License

\begin{abstract}
ZAMBIA'S nation-wide adult Human Immunodeficiency virus (HIV) prevalence stood at 13.3 percent in 2014. In Southern Province, 12.8 percent of adult men and women aged 15-49 years were HIV positive. The prevalence was higher in women than in men (15.1\% compared to $11.3 \%$ ). The most recent data also suggested that key populations were disproportionately infected with HIV compared to the general population. The Measure Know Your Response (KYR) study took place from September 2013 to July 2014 in the Southern Province of Zambia. The aim was to map and characterize the HIV response among both government (health and none health) and non-government organizations (NGOs). The purpose was to identify gaps in the response, in particular in terms of the geographical areas and the key populations attended to and those disproportionately missed. This paper focuses on the results from the mapping of NGOs involved in HIV and AIDS response. For this aspect of the mapping data were collected using the NGO Sector Questionnaire. Focus was interventions of NGOs and their services to target populations across all the 12 districts of the Southern Province. All program implementers working in a given district were asked questions to assess the extent to which their HIV-prevention activities in the district included strategies to address all key populations, including gender issues. Data from the quantitative questionnaires was analyzed using STATA version 12.0 and Microsoft Excel. The results of the pilot test revealed gaps in both geographic spread and content of programs in the NGOs run HIV-prevention response. For instance, hardly any NGOs worked in the new and smaller districts of Pemba, Siavonga, and Zimba. Similarly, certain services, such as mass media campaigns, interpersonal education and persuasion programs were being implemented across all the districts. Further, fewer than three of the 93 organizations surveyed targeted key populations, notably sex workers, incarcerated populations, men who have sex with men (MSM), transgender people and injective drug
\end{abstract}

users. With respect to gender, about $25 \%$ of the organizations did not integrate gender issues into their HIV-prevention activities. From a methodological standpoint, the pilot test revealed the need to examine dimensions of program content, quality and intensity. In spite of these limitations, the study permits us to know which interventions are being implemented by the NGO sector, contributing to new knowledge in Zambia about geographic gaps in the availability of HIV-prevention interventions.

\section{Keywords HIV Prevention, Zambia, NGO Interventions, Key Populations}

\section{Introduction}

Zambia's HIV epidemic is now characterized as mature and generalized, as opposed to being concentrated in specific populations [1]. Most HIV transmission primarily occurs heterosexually. The spectrum estimates of the HIV prevalence in adults aged 15-49 years show that the HIV pandemic has been fairly stable over the last 15 years, after the initial peak prevalence of around 27\% in the 1990s [1]. A very modest decline has been noted over the years.

According to the Demographic and Health Survey (DHS), the adult HIV prevalence rate stood at 13.3 percent in 2014, with prevalence of $15.1 \%$ in women higher compared to $11.3 \%$ in men [2] In Southern Province where this study was conducted, 12.8 percent of adult men and women aged 15-49 years were HIV positive, making it the fourth highest among the 10 provinces of Zambia[1]. At district level, comparison of data from NAC showed that in 2010, HIV prevalence was highest in Livingstone (25.3 percent) followed by Mazabuka (18.4 percent). Overall, 15.7 percent of adults in the districts of Choma, Monze and Siavonga and 15.2 percent of those in Kalomo and Kazungula were HIV positive. The most rural districts 
in the province, Gwembe and Namwala had the lowest (6.2\%) prevalence of HIV in Southern Province compared to more urbanized districts such as Livingstone, Monze, Choma and Mazabuka. The DHS data also showed that HIV prevalence increased with wealth quintile and level of education. [2].

The Revised National AIDS Strategic Framework 2014-2016 [1] identifies the sources of new infections of $\mathrm{HIV}$ in Zambia and is strengthening response strategies to deal with them. These are: multiple and concurrent sexual partners, low and inconsistent condom use, mobility and labor migration, low levels of male circumcision, vertical transmission from mother-to-child, high risk behaviors among sex workers and in male-to-male sexual relationships. Other factors that increase vulnerability to HIV infection include alcohol abuse, gender inequality, gender-based violence and sexual coercion, inter-generational and transactional sex as well as taboos and barriers regarding communication about sex among couples [1]. Underlying many of these factors are structural issues such as social and cultural norms, negative legal environment as well as religious and social stigma.

Over the past 30 years that Zambia has been grappling with managing the pandemic, the country has developed and implemented a wide range of programs across the four pillars as defined in the 2011 - 2015 National AIDS Strategic Framework. The four pillars are: Prevention; Mitigation; Treatment and Care; as well as, Management and Coordination [1]. The focus was to prevent new infections and improve the quality of life of those infected and affected by HIV and AIDS. The Revised National HIV and AIDS Strategic Framework of 2014-2016 identified three national priorities for the HIV and AIDS national response:

a To take to scale and sustain the gains made in high-impact interventions (basic programs which include HIV testing and counselling, antiretroviral therapy, condom programming, voluntary medical male circumcision, prevention of mother to child transmission of HIV and social behavioral change communication);

b To ensure that the critical enablers such as gender equality, legal and policies environment, political commitment and governance, resource mobilization, coordination and management of the response provide an enabling environment for the response; and,

c To synergize with other development sectors such as health, education, social protection, poverty alleviation and livelihood, food security and nutrition, health System strengthening, HIV and AIDS in the work place, community system strengthening, HIV and AIDS mainstreaming in capital projects for efficiency and effectiveness.

\section{Literature Review}

Studies on the role of non-governmental organisations (NGOs) in HIV prevention have been carried out in many parts of the world. In Central and Eastern Europe and Central Asia, Directors of 29 Non-governmental organisations operating in countries that were participating in the Global AIDS Intervention Network (GAIN) project participated in a study aimed at evaluating the effectiveness of computer-based methods for improving information exchange and technology transfer between HIV-prevention researchers and international service providers.[3p13]. The study used in-depth semi-structured interviews to gather systematic information about NGOs organizational characteristics, budgets, funding sources, and sources of information used for program development; the types of HIV prevention programs carried out as well as populations served by NGOs in the region; and barriers faced by the organizations. In addition, the study provided detailed information about innovative prevention programs that appeared effective and were well-received in the NGOs' communities. [3]. Results of the study show that most of the common types of prevention activities carried out by the NGOs were needle exchange, peer education, and public awareness activities such as distributing educational materials. Among the major barriers identified by the study to be hampering the effective conduct of HIV prevention programs were a shortage of available financial resources at 72 percent, governmental indifference or opposition at 59 percent, and AIDS-related stigma at 31 percent. The study also established that national governments in the surveyed countries rarely provided substantial funds for NGO programs, and that most funding came from United Nations agencies or private foundations. [3 pp13-14].

A similar study covering 23 countries that were participating in the GAIN project was carried in Latin America and the Caribbean [4 p155]. The purpose of this study was to obtain detailed information about prevention programs that were being carried in Latin America and Caribbean. [4 p155]. Findings of the study were that the 23 NGOs that participated in the study had conducted 58 direct-service programs and had also been carrying HIV prevention activities for a mean of 8 years. Further, findings show that many of the NGOs had relied on volunteers to carry out their HIV prevention activities. Activities conducted by NGOs included train-the-trainer activities at 43.5 percent and face-to-face prevention activities at 34.8 percent. The study also established that the main obstacles faced by the NGOs were lack of funding at 60.9 percent and the fear of HIV-related stigma and discrimination at 56.5 percent while religious, cultural and secular beliefs regarding condom use, homosexuality, and AIDS were at 43.4 percent. [4 p157].

On the African continent Rau identified the strengths 
and the challenges of civil society organizations. [5 p285]. The author identified political factors as the ones responsible for placing NGOs at the forefront the provision of HIV prevention interventions particularly among African countries. On the other hand, however, the author also claims that political factors are guilty of rewarding the achievements of NGOs with little more than lip service from international and national HIV/AIDS agencies. [5 p285]. While acknowledging that family and community members in general provide extensive care and support to those in need, Rau identifies women's support groups, religious communities and savings societies as important organizing mechanisms to assist people living with HIV (PLWH), caregivers and orphaned children. [5 p286] Within the African context, another study investigated the role played by NGOs in preventing HIV as well as supporting the infected people. In this study, 72 respondents from two of the oldest NGOs involved in HIV prevention in Tanzania were asked to mention some of the services that they provided. Findings reveled that, NGOs in Tanzania provided services such as voluntary counselling and testing, HIV education, medical services, home based care and many others that contribute to preventing the spread of HIV. [6].

In South Africa, a study was carried out in rural parts of the Eastern Cape Province to assess the impact of Stepping Stones, an HIV prevention program implemented by the South African Planned Parenthood Association. [7 pp1-2]. The Stepping Stones program was originally developed in Uganda in 1995 as a participatory HIV prevention program that aims to improve sexual health through building stronger, more gender equitable relationships [7 pp1-2]. Findings of this study were that while the Stepping Stones project did not lower the HIV incidences, it had a positive impact on several risk factors for HIV notably, HSV-2 and perpetration of intimate partner violence. [7 $\mathrm{p} 7$ ].

In Zambia, similar studies have been carried out but most significant to the present paper is the study that was carried out in 1998 to evaluate peer education programs of HIV and AIDS projects ran by four non-governmental organizations.[8 p398] The programs were assessed only in terms of immediate developmental outcomes to target groups rather than on long-term impact in the area of peer education, reduction in transmission of HIV, helping communities to cope with those infected, and to maintain human dignity and human rights.[8 p398]. Findings of the study were that the peer education programs had raised community awareness of HIV and AIDS and that basic knowledge of HIV and AIDS was, for the most part, accurate. The study also established evidence that due to the intervention of the programs, certain traditional practices, which contributed to the spread of the virus (e.g. sexual cleansing and scarification), were becoming modified over time. Further, the study established that the programs were also instrumental in protecting human rights and enabling people living with HIV and AIDS to maintain their dignity. [8 pp401-05].

Past studies have sought to establish the role of NGOs in the prevention of HIV. However, the present study will present the characteristics of NGOs that were involved in HIV prevention and the types of responses that these organizations provided in Southern Province, Zambia. This focus is central to programing selection and priorities of HIV prevention activities because NGOs are a major government partner, they bring to the field the much-needed resources and technical assistance.

\section{Objectives of the Study}

The overall objective of this study was to generate evidence to inform the selection and prioritization of evidence based and high impact interventions on HIV prevention and incidence in Zambia.

The specific objectives of the study were three-fold as follows:

1. Profile and characterize the program implementers;

2. Determine what specific HIV-prevention interventions were being implemented and what resources were available to implement them; and,

3. Determine and characterize the gender mainstreaming into HIV prevention programs.

\section{Methods Used for Mapping NGO Interventions}

The data come from a MEASURE Evaluation-supported pilot test of the Know Your Response (KYR) toolkit which was implemented from September to October 2013 in Southern Province, Zambia. The study was implemented jointly by Tulane University in the United States of America and the Institute of Economic and Social Research (INESOR) at University of Zambia, in collaboration with the National HIV/AIDS/STI/TB Council (NAC). The specific objectives of the pilot test were to: (a) identify and eliminate problems in the KYR tools and enable corrective changes or adjustments to be made; (b) permit a thorough test of the logistical arrangements and planned statistical as well as analytic procedures; and (c) determine whether data collected via the tools yielded information that was needed to assess the extent to which prevention interventions were focused on geographic areas where HIV was spreading most rapidly, and covered technical recommendations for HIV-prevention among populations at higher risk of HIV exposure. An additional objective was to share the results of the pilot test with key stakeholders and data users to determine whether the tools provided data needed to answer policy and programmatic questions. 
The study had five instruments for data collection as follows:

1. Policy Checklist

2. Strategic Information Checklist

3. Program Implementers Core Questionnaire

4. Program Implementers Modules for Key Populations at Higher Risk of HIV Exposure (and other vulnerable populations)

5. District Health Questionnaires, which was composed of the following modules:

i. Module 1: Health Facility

ii. Module 2: Government non-Health Sector HIV Prevention Programs

The tool kit addresses three key concepts that are critical for an improved understanding of the HIV-prevention response - location, scale, and needs and helps to provide answers to the following pertinent questions: Who is doing what and where? Who is being reached by HIV-prevention programs? Are the needs of key populations at higher risk of HIV exposure being met in settings where they are located? Therefore, the KYR tools permit a gap analysis, geographic mapping of the current HIV-prevention response, and provide information needed at the national and sub-national levels to guide the adjustment and prioritization of HIV-prevention programs and interventions, with a view to delivering prevention services to peoples and places where they are most needed. The tools were an adaptation of several existing tools, including WHO's Prevention Service Availability Mapping (PSAM); the UNAID's Policy \& Strategic Information Checklists; tools used in a situational analysis of response to HIV in Belize; the HIV Policy Assessment Tool, and United Nations Population Fund (UNFPA) Rapid Assessment Tool for Sexual and Reproductive Health and HIV Integration

This paper focuses on the Program Implementers Core Questionnaire. The Program Implementer Core Questionnaire was administered to all HIV-implementing organizations operating in the province based on a list of registered implementers provided by the NAC. A total of 93 Program Implementers in the non-governmental sector were successfully interviewed (Table 1). These included non-governmental organizations (NGOs), faith-based organizations (FBOs), civil society organization (CSOs), the private sector, etc.

The Core Questionnaire collected basic information about the implementing organization. For each organization that currently conducted HIV-prevention activities, data were collected on the type of HIV-prevention activities implemented in the past 12 months, financial resources expended for HIV prevention activities in the past 12 months, and sources of funding for HIV-prevention activities implemented in the past 12 months. In addition, the Core Questionnaire collected information on the number of people reached with HIV-prevention activities by the organization, by district of residence and by sex.

The structure of the Core Questionnaire was guided by Michael Sweat's framework for classifying HIV-prevention interventions, published by UNAIDS. [9]. Information was collected on six categories of interventions:

i. Standardized hybrid interventions commonly used (e.g., VCT, social mobilization, comprehensive sex education);

ii. Interventions affecting knowledge, attitudes and beliefs \& influencing psychological \& social risk correlates (e.g., mass media campaigns, prevention counselling, etc.);

iii. Harm reduction interventions (e.g., condom distribution, needle and syringe exchange, etc.);

iv. Biological/biomedical interventions that reduce HIV-infection \& transmission risk (e.g., post-exposure prophylaxis, male circumcision, etc.);

v. Mitigation of barriers to prevention and negative social outcomes of HIV infection (e.g., training of service providers and law enforcement officers, etc.); and,

vi. Mitigation of biological outcomes of HIV infection (e.g., HIV/tuberculosis (TB) treatment services, opportunistic infection prophylaxis, palliative care, etc.).

\section{Gender Integration}

All program implementers working in a given district were also asked questions to assess the extent to which their HIV-prevention activities in the district included strategies to address gender issues and the type of gender issues addressed. Program implementers were also requested to provide a brief description of the activities implemented to address the specified gender issues. Response categories for the extent to which gender was integrated into HIV-prevention activities ranged from 4 to 1 and were as follows: 4. addresses all the key gender issues; 3. addresses most of the key gender issues; 2. addresses some key gender issues; and 1. did not address key gender issues.

A multiple-response question inquired as to whether the following gender issues were addressed: (a) Gender norms; (b) Violence against women and girls; (c) Cross-generational sex; (d) Gender-related barriers to access and utilization of HIV/AIDS services; (e) Gender differentials in caregiving for HIV-infected and affected people; (f) Gender differentials in access to education and economic security; and, (g) Other. In a subsequent open-ended question, organizations were asked to briefly describe the activities they had implemented to address the gender issues specified.

The study methodology consisted of the following stages: 
1. Administering the Questionnaire Appraisal System (QAS) Form to NAC staff: The QAS is a systematic appraisal of survey questions and helps to spot potential problems in the wording or structure of the questions that may lead to difficulties in question administration, miscommunication, or other failings. A meeting was organized with NAC staff during which they examined each question in the data collection instruments by considering question characteristics in a step-wise fashion. At each step, a decision was made as to whether the question exhibited features that were likely to cause problems and suggestions were made as to how the questions were to be rephrased.

2. Cognitive interviewing using the active probing approach: The purpose of cognitive interviewing was to look at question answering from the respondent's perspective. This approach helped the researchers to understand cognitive strategies used to answer a question and the ways in which a question performed across different respondents. This approach helped to highlight question design problems and provided insights into incorrect interpretations of questions, so as to suggest possible revisions to the questions.

3. Updating of the list of current HIV and AIDS implementing organizations in Southern Province: Using the list provided by the NAC as a starting point, a snowball method was used to ask known implementing partners to direct the research team to other organizations working on HIV/AIDS prevention in the province and/ or district but which were not captured on existing directories/lists.

4. Testing data collection methodologies to determine which one works best: Districts in Southern Province of Zambia were randomly assigned to one of the following groups: (a) face-to-face interviews whereby the interviewer was to read out all questions to the respondent and fill in the responses provided; and (b) self-administered questionnaire filled out in the presence of an interviewer, whereby the respondent was to complete the questionnaire by him or herself but could ask the interview clarifying questions.

5. Conducting focus group discussions in order to detect potential problems with the data collection tools and improve them.

The study was approved by the Tulane University Human Research Protection Program Biomedical Institutional Review Board, New Orleans, Louisiana. Local IRB approval was obtained from ERES Converge and authorization to conduct the study from the Zambia
NAC and the country's Ministry of Health. Interviewers and supervisors received a one-day research ethics training based on the Family Health International 360 Research Ethics Curriculum. The data collection instruments were pretested on HIV program implementers in Chongwe and Kafue districts near Lusaka in July 2013. The data were analyzed using STATA version 12.0. To estimate implementation rates for each intervention in a given district, percentages were calculated based on the total number of organizations that were completely interviewed (i.e., $\mathrm{N}=93$ program implementing organizations for each calculation and cell of a table).

\section{Results of the Study}

\section{Characteristics of Program Implementers}

The NAC 2011-2012 data base had identified 111 registered program-implementing organizations in ten districts of Southern Province. It is to be noted that Pemba and Zimba were new districts. At the time the list of registered NGOs was created, Pemba was under Choma District and Zimba was part of Kalomo and Kazungula districts. Assuming that all registered organizations operating in Choma (a total of 15) also worked in Pemba and all registered organizations operating in Kalomo and Kazungula (a total of 10) also worked in Zimba, the total number of registered NGOs in Southern Province at the time of the study was 132, yielding a response rate of $84.1 \%$. This is a rough approximation as some NGOs on the list could not be located, and the list was also not up-to-date.

Table 1 (below) shows that the 93 NGOs involved in HIV prevention in Southern Province had carried out a total of 152 activities in the 12 months preceding this study

Table 1. Numbers of organizations surveyed in Southern Province and HIV-prevention activities they implemented in 2013 by district $(\mathrm{N}=93)$

\begin{tabular}{|c|c|c|c|}
\hline District & $\begin{array}{c}\text { Number } \\
\text { of NGOs }\end{array}$ & $\begin{array}{c}\text { Proportion to total } \\
\text { number of NGOs } \\
\text { (\%) }\end{array}$ & $\begin{array}{c}\text { Number of } \\
\text { activities }\end{array}$ \\
\hline Choma & 15 & 16.1 & 26 \\
\hline Gwembe & 4 & 4.3 & 3 \\
\hline Kalomo & 8 & 8.6 & 8 \\
\hline Kazungula & 2 & 2.1 & 3 \\
\hline Livingstone & 18 & 19.3 & 39 \\
\hline Mazabuka & 19 & 20.4 & 15 \\
\hline Monze & 11 & 11.8 & 9 \\
\hline Namwala & 8 & 8.6 & 8 \\
\hline Pemba & 1 & 1.1 & 9 \\
\hline Siavonga & 2 & 2.1 & $\mathbf{1 5 2}$ \\
\hline Sinazongwe & 4 & 4.3 & 1.1 \\
\hline Zimba & 1 & $\mathbf{1 0 0}$ & \\
\hline Total & $\mathbf{9 3}$ & & 29 \\
\hline
\end{tabular}


Table 1 further shows that there were geographic disparities in the availability of NGOs implementing HIV-prevention activities in different districts of the province. The majority, 19-20 percent, of organizations surveyed were conducting HIV-prevention activities in Livingstone and Mazabuka and respectively. This group was followed by 16 percent of organizations that were working in Choma, while the lowest number, representing one percent each were operating in Pemba and Zimba.

In terms of funding sources, most program implementers received funding for HIV-prevention from non-governmental sources, with slightly more than half $(54 \%)$ obtaining funding from specified donors. One in every five program implementers obtained funding for HIV-prevention activities from other non-governmental, faith-based or community-based organizations. Only three percent of organizations surveyed received funding from the private commercial sector. Government funding for HIV-prevention was reported by 6 percent of organizations surveyed.

\section{HIV Prevention Interventions by NGOs}

The following sections present the interventions that were reported to have been implemented during the 12-month period under study.

\section{Standardized Interventions in Common Use}

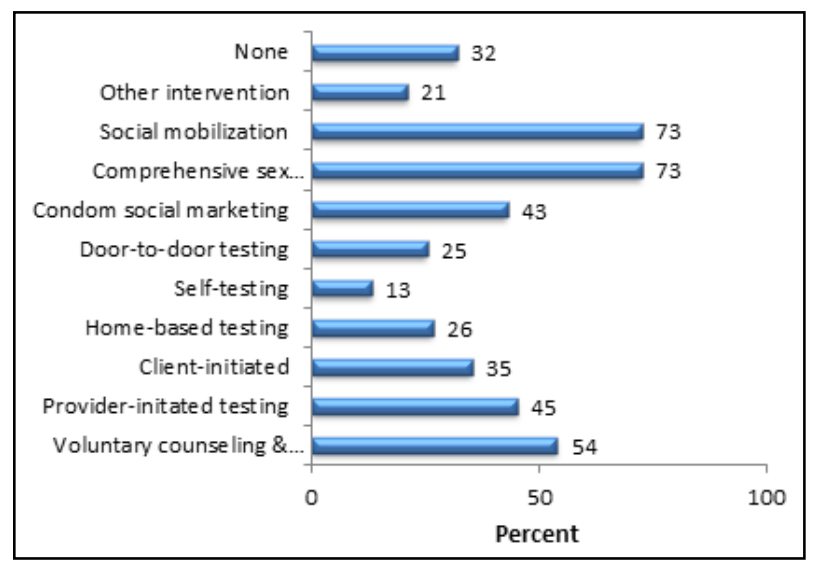

Figure 1. Percentage of organizations surveyed in Southern Province that were implementing standard HIV-prevention interventions by type of intervention, Zambia 2013.

According to Sweat's framework, standardized hybrid interventions commonly used include voluntary counselling and testing (VCT), condom social marketing, comprehensive sex education, and social mobilization. [9].The clear majority of organizations surveyed in Southern Province were currently implementing at least one of these interventions. As shown in Figure 1, the interventions most commonly implemented were social mobilization and comprehensive sex education and were each reported by 73 percent of organizations surveyed.
More than half of organizations surveyed provided VCT, while 45 percent reported provider-initiated testing services as compared to 13 percent that said they provided self-testing services. Figure 1 further shows that door-to-door and home-based testing activities were reported by nearly one in every four organizations surveyed.

There were geographic disparities in the availability of standardized interventions which reflect, in part, the distribution of program implementers by district. None of the organizations surveyed were implementing condom social marketing, comprehensive sex/HIV education, and social mobilization activities in Pemba. In addition, none of the organizations surveyed were implementing social mobilization activities in Siavonga and condom social marketing interventions in Sinazongwe. Choma had the largest concentration of organizations providing VCT and condom social marketing interventions. Choma, Livingstone and Mazabuka had the largest concentration of organizations providing comprehensive sex/HIV education and social mobilization interventions. The data also show rural-urban disparities in the availability of standard interventions within districts of Southern Province. None of the NGOs surveyed were implementing VCT, condom social marketing, comprehensive sex/HIV education and social mobilization interventions in urban areas of Gwembe and Kazungula. In Livingstone, Mazabuka and Choma, more NGOs provided VCT, condom social marking, comprehensive sex/HIV education, and social mobilization in urban than in rural areas.

\section{Interventions Affecting Knowledge, Attitudes and Beliefs}

Interventions affecting knowledge, attitudes and beliefs as well as those influencing psychological and social risk correlates include mass media campaigns; interpersonal education and persuasion programs, face-to-face dialogue; sex education; education to promote adherence to universal precautions; and, prevention counselling. The clear majority of NGOs implemented at least one of these interventions, with the least common being mass media campaigns (30 percent of NGOs surveyed). In three districts - Pemba, Siavonga, and Zimba - none of the organizations surveyed implemented mass media campaigns, interpersonal education and persuasion programs. In addition, no organization surveyed implemented sex education and prevention counselling programs in Pemba district.

The data also show some rural-urban disparities in NGO implementation of interventions affecting knowledge, attitudes, and beliefs. No organization surveyed in Southern Province implemented interventions affecting knowledge, attitudes and beliefs in the past 12 months in urban areas of Kazungula and Gwembe, the only exception in the latter district being prevention counseling. Rural-urban disparities were observed in Choma, Kalomo, 
and Livingstone, with more of the NGOs surveyed implementing interpersonal education/persuasion programs and sex education programs in urban than in rural areas. Rural-urban disparities were also seen in the percentage of NGOs surveyed that implemented prevention counselling in Livingstone in the past 12 months ( 8 percent in rural areas versus 15 percent in urban areas). Also worthy of note is the presence in rural Sinazongwe and absence in urban areas of the district of NGOs implementing mass media campaigns and interpersonal education and persuasion programs.

\section{Harm Reduction Interventions}

Harm reduction interventions are defined as interventions that lower the risk of a given behavior but do not eliminate the behavior. For the purposes of this study, harm reduction interventions included: distribution of condoms and condom-compatible lubricants, needle and syringe exchange, provision of equipment required for universal precautions, providing safe spaces for vulnerable populations to use prevention services and inject drugs safely and livelihood alternatives to transactional sex. The most common harm reduction intervention was distribution of condoms, which was implemented by 63 percent of organizations surveyed. It is to be noted, however, that many of the organizations did not distribute condom-compatible lubricants and we deleted this aspect of the intervention from the questionnaire. The least common harm reduction interventions were needle and syringe exchange, which probably reflected the fact that injecting drug use was not a key driver of the HIV epidemic in Zambia and is illegal, and livelihood alternatives to transactional sex.

There was at least one surveyed organization involved in the distribution of condoms and condom-compatible lubricants in each district, except in Zimba. Needle and syringe exchange were reported as being implemented in each district by at least one organization surveyed, the exceptions being Pemba and Siavonga, which also had no organizations that provided equipment required for universal precautions or safe spaces for vulnerable populations to use prevention services or inject drugs safely. The latter intervention was the least common and was reported as being implemented by organizations surveyed in only half of the districts of Southern Province. No organization surveyed implemented livelihood alternatives to transactional sex in Gwembe, Pemba and Sinazongwe.
Rural-urban gaps in the implementation of harm reduction interventions are revealed by the data. Condom distribution was reported as being implemented by at least one organization surveyed in the rural and urban areas of each district, except for urban Gwembe, urban Kazungula, urban Pemba and both rural and urban areas of Zimba. In three districts, no organization reported implementing rural needle and syringe exchanges programs: Choma, Pemba, and Siavonga. For this intervention, gaps are also seen in the provision of this intervention in urban areas of three districts: Gwembe, Kazungula, Pemba, and Siavonga. Few organizations surveyed reported that they provided equipment required for universal precautions in the past 12 months. No organization surveyed reported providing this intervention in rural Choma, rural Monze, rural Pemba, rural Siavonga, and rural Zimba.

There was also an absence in the sample of organizations that provided equipment required for universal precautions in urban areas of Gwembe, Kazungula, Pemba, Siavonga, and Zimba. In both rural and urban areas, the provision of safe spaces for vulnerable populations to use prevention services and inject drugs safely was the least common intervention offered in the past 12 months by organizations surveyed. Regarding the provision of livelihood alternatives to transactional sex, there were five districts in which no organization surveyed reported implementing this intervention in urban areas: Choma, Gwembe, Kazungula, Pemba and Siavonga.

\section{Biological and Biomedical Interventions}

Zambia is promoting and implementing an integrated package of interventions that includes biological and biomedical (BB) interventions to reduce HIV-infection and transmission risk. To assess the prevalence of the $\mathrm{BB}$ interventions, organizations that participated in the study were asked to indicate the BB interventions they were offering in each district. Table 2 shows that in 2013, the most common BB interventions were STI diagnosis and treatment and breastfeeding substitution for infants born to HIV+ mothers, which were being implemented by various organizations in eight out of the twelve districts. Family planning services and post-exposure prophylaxis were also common, being offered by the same organizations in seven out of the twelve districts. The least implemented BB interventions were ARV prophylaxis for infants born to HIV+ mothers and male circumcision, which were implemented in five and four out of the twelve districts, respectively. 
Know Your Response: Pilot Mapping of HIV Prevention Responses by

Non-governmental Organizations in Southern Province, Zambia

Table 2. Biological or Biomedical Interventions Implemented in Southern Province, Zambia in 2013

\begin{tabular}{|c|c|c|c|c|c|c|}
\hline District & $\begin{array}{l}\text { STI Diagnosis } \\
\& \text { Treatment }\end{array}$ & $\begin{array}{l}\text { Post-exposure } \\
\text { Prophylaxis }\end{array}$ & $\begin{array}{c}\text { Family } \\
\text { Planning }\end{array}$ & $\begin{array}{c}\text { Male } \\
\text { Circumcision }\end{array}$ & $\begin{array}{c}\text { ARV Prophylaxis } \\
\text { for Infants Born to } \\
\text { HIV+ Mothers }\end{array}$ & $\begin{array}{l}\text { Breastfeeding } \\
\text { Substitution for } \\
\text { HIV+ Mothers }\end{array}$ \\
\hline Choma & Yes & Yes & Yes & Yes & Yes & Yes \\
\hline Gwembe & Yes & Yes & Yes & Yes & Yes & Yes \\
\hline Kalomo & Yes & Yes & Yes & Yes & No & Yes \\
\hline Kazungula & Yes & Yes & Yes & No & No & No \\
\hline Livingstone & Yes & Yes & Yes & No & Yes & Yes \\
\hline Mazabuka & Yes & Yes & Yes & No & Yes & Yes \\
\hline Monze & Yes & Yes & Yes & Yes & Yes & Yes \\
\hline Namwala & Yes & No & No & No & No & No \\
\hline Pemba & No & No & No & No & No & No \\
\hline Siavonga & No & No & No & No & No & Yes \\
\hline Sinazongwe & No & No & No & No & No & Yes \\
\hline Zimba & No & No & No & No & No & No \\
\hline
\end{tabular}

The data further shows geographical differentials in the distribution of available selected biomedical/biological interventions. For example, two of the districts Choma and Gwembe had all the selected BB interventions being implemented by the surveyed organizations while only one of the selected BB interventions was being offered by the surveyed organizations in five of the districts Namwala, Siavonga and Sinazongwe. In Namwala, the only biological/biomedical intervention offered by organizations surveyed was diagnosis and treatment of sexually-transmitted infections. It can also be observed from Table 2 that there were no $\mathrm{BB}$ interventions implemented in the newly created districts of Pemba and Zimba. These geographical differentials points to inequities in provision of health services across the districts of the Southern Province.

Qualitative data showed that biological and biomedical interventions that reduce HIV-infection and transmission risk were not commonly reported by the organizations visited. Most of the organizations were mainly involved in health education in both rural and urban areas. However, Family Health International and Corridors of Hope were among organizations that were directly linked to such interventions, particularly to male circumcision, family planning, and ARV prophylaxis. The organizations implementing breastfeeding substitution for HIV + mothers were present in eight districts while those implementing family planning and post exposure prophylaxis were equally present in seven districts respectively. It can also be noted that only four districts had organizations promoting voluntary medical male circumcision Namwala, Gwembe, Choma and Monze.

\section{ARV Prophylaxis for Infants Born to HIV+ Mothers}

In Choma, the organizations visited reported that most health facilities were mentored in the implementation of option $\mathrm{B}+$ for the elimination of mother-to-child transmission, in addition to the prophylaxis given to the exposed infants. They stated that the ministry was moving towards initiating all positive pregnant mothers on ART. In view of this, most organizations incorporated such interventions to reach the goal. In addition, one organization, Corridors of Hope, stated that it did not only focus on prophylaxis given to the exposed infants, but also on the follow up of the infants and the mothers. It was noted that Corridors of Hope trained health facility staff to do virology test of infants at different ages to reduce the risk of transmission. It was reported that the plan was to roll out the intervention to all the districts but due to human resource and logistical challenges, only few districts were selected.

\section{Voluntary Medical Male Circumcision}

Voluntary medical male circumcision was reported to have been implemented only in four districts as shown in the table. The organizations mainly involved were Family 
Health International and Corridors of Hope. The organizations reported that the demand for voluntary medical male circumcision (VMMC) was generally low in the province. One of the program managers interviewed stated that despite the province having a high HIV prevalence rate in the country, the demand for VMMC was quite low. It was reported that there were myths surrounding the intervention and that NGOs were working on sensitizing the districts. One important aspect which was highlighted was that the demand was more during campaign months such as April, August and December. It was also noticed that inadequate male circumcision providers and logistics were some of the factors which led other organizations not to implement the intervention. World vision and Family health international supported static sites in the urban areas while rural areas were serviced through mobile and outreach services.

\section{Family Planning}

Family planning was reported in 7 districts as shown in the table below, most of the organizations reported that there was significant progress towards $\mathrm{HIV}+$ clients accepting family planning methods. However, it was also noted that condoms were preferred to other long-term methods. In Choma, World Vision reported that, as the number of new family planning acceptors was quite low in the rural areas, most of the organization's activities were geared to creating demand for family planning services.

\section{STI Diagnosis and Treatment}

Organizations such as NZP+ also promoted diagnosis and treatment of sexually transmitted infections (STIs) in Livingstone and Choma. Other organizations involved in STI diagnosis stated that they trained community volunteers and lay counsellors who made follow ups of clients and their partners in the community. However, some organizations reported that in rural health facilities, guidelines on treatment and management of STIs were not present hence staff needed to be trained to offer a complete package of STI management and treatment services. In addition, most of the STI clients were not tested for HIV due to a shortage of test kits in some facilities. The STI cases were reported to be prevalent among school-going children, mostly girls who presented with vaginal discharge. The Anti-AIDS teachers' associations were actively involved in providing health education to pupils so as to mitigate the spread. It was reported that the associations had plans to distribute condoms to schools but most of the schools were unsupportive.

Table 3. Interventions to Mitigate Biological outcomes of HIV in Southern Province, Zambia in 2013

\begin{tabular}{|c|c|c|c|c|c|}
\hline District & $\begin{array}{c}\text { Tuberculosis } \\
\text { Prevention, Diagnosis } \\
\text { \& Treatment }\end{array}$ & $\begin{array}{c}\text { HIV Treatment } \\
\text { with Antiretroviral } \\
\text { Drugs }\end{array}$ & $\begin{array}{c}\text { HIV-related } \\
\text { Opportun-istic Infection } \\
\text { Prophylaxis and } \\
\text { Treatment }\end{array}$ & $\begin{array}{c}\text { Prevention, } \\
\text { Diagnosis and } \\
\text { Treatment of Viral } \\
\text { Hepatitis }\end{array}$ & $\begin{array}{l}\text { Palliative Care } \\
\text { for People } \\
\text { Living with HIV }\end{array}$ \\
\hline Choma & No & Yes & No & No & Yes \\
\hline Gwembe & Yes & Yes & Yes & No & Yes \\
\hline Kalomo & Yes & Yes & No & Yes & Yes \\
\hline Kazungula & No & No & No & No & No \\
\hline $\begin{array}{c}\text { Livingston } \\
\mathrm{e}\end{array}$ & No & Yes & Yes & Yes & Yes \\
\hline Mazabuka & Yes & No & Yes & No & Yes \\
\hline Monze & No & No & No & No & Yes \\
\hline Namwala & No & No & No & Yes & Yes \\
\hline Pemba & No & No & No & No & No \\
\hline Siavonga & No & No & Yes & No & Yes \\
\hline $\begin{array}{l}\text { Sinazong } \\
\text { we }\end{array}$ & No & No & No & No & Yes \\
\hline Zimba & No & No & No & No & Yes \\
\hline
\end{tabular}




\section{Interventions to Mitigate Biological Outcomes of HIV Infection}

The biological outcomes of HIV and AIDS are devastating. To mitigate these outcomes of HIV infection, several interventions include: (a) TB prevention, diagnosis and treatment services; (b) HIV treatment with antiretroviral (ARV) drugs; (c) HIV-related opportunistic infection prophylaxis and treatment; (d) prevention, diagnosis and treatment of viral hepatitis (allowing access to antiretroviral treatment); and (e) palliative care for PLWH. Table 3 indicates that the most common intervention to mitigate biological outcomes of HIV infections was palliative care for PLWH, which was being implemented by the surveyed organizations in ten out of the twelve districts. Each of the other interventions was implemented in at least three out of the twelve districts.

The data shows that none of the interventions to mitigate biological outcomes of HIV infection was implemented in Kazungula and Pemba by organizations surveyed. In Gwembe, Kalomo and Livingstone, four of the five interventions shown were implemented by at least one organization surveyed but the districts differed in terms of which intervention was not provided. In Gwembe, no organization surveyed implemented interventions to prevent, diagnose and treat viral hepatitis. In Kalomo, it was only HIV-related opportunistic infection prophylaxis and treatment that was not provided by any of the organizations surveyed.

Insights from qualitative data reveal that programs and activities to mitigate the biological outcomes were integrated with other activities as reported by the organizations surveyed. Some organizations such as World Vision worked with the community in sensitization of TB among HIV+ clients. The TB treatment supporters were trained in various health facility catchment and were therefore able to collect sputum and send to the nearest laboratory. All the smear positive clients were immediately commenced on treatment. In view of this, there seemed to be an improvement in the TB cure rate among HIV+ positive clients. It was also reported there was an increase in the TB detection rate. In addition, some smear positive clients were also subjected to HIV test and those who were found HIV+ were further assessed for ART eligibility and consequently initiated on the treatment. The program managers interviewed stated that they worked closely with the Health departments to successfully implement the activities.

The Network of People Living with HIV (NZP+) reported having implemented activities about palliative care for people leaving with HIV. It was noted that the existence of the organization seemed to have contributed in the reduction of stigma and discrimination in the province. The HIV work place policy was also promoted in all the work places and most of the workers and the employers were oriented in the period under review. In addition, it was reported that HIV+ clients who were in denial were counselled so that they could be initiated on Aural Retroviral Treatment (ART) while those currently on ART but had defaulted were followed up. However, it was reported that such activities were demanding and needed human resource and funds to be effectively carried out.

\section{Interventions to Mitigate Barriers to Prevention and Negative Social Outcomes of HIV Infection}

The Zambian government's response to HIV and AIDS revolves around four pillars of prevention, treatment, care and support, impact mitigation and response management. Prevention, care and support, impact mitigation as well as response management are key interventions which program implementers from the non-government sector could provide. These have been grouped as interventions to mitigate barriers to prevention and negative social outcomes of HIV infection. This group of interventions typically includes training of service providers and law enforcement; separate accommodation to protect at-risk population; self-help and solidarity groups; financial and in-kind sustenance support; medical and legal assistance services; counselling; legal, policy and institutional reform to protect human rights of vulnerable groups and PLWH. Most organizations (82 percent) implemented counselling in the past 12 months. More than half of the organizations surveyed trained service providers and law enforcement agencies while three in every ten worked on legal, policy and institutional reforms. The provision of medical and legal assistance services and separate accommodation to protect at-risk populations were uncommon interventions and were implemented by 18 percent and two percent of organizations, respectively. While three selected interventions, namely training service providers and law enforcement agencies; self-help and solidarity groups as well as financial and in-kind sustenance support were provided in seven of the districts, no service provider surveyed provided any of this support to PLWH in Pemba and Siavonga

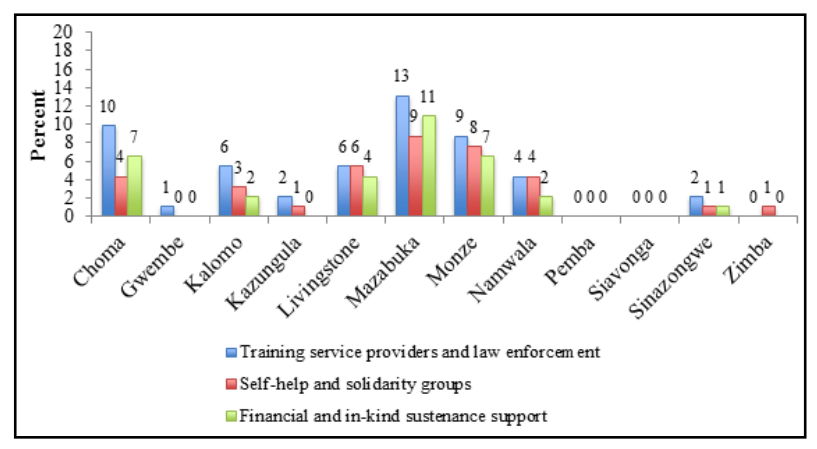

Figure 2. Percentage of surveyed organizations that implemented interventions to mitigate barriers to prevention and negative social outcomes of HIV infection in Southern Province, Zambia in 2013.

With the additional exception of Gwembe, at least one organization surveyed promoted self-help and solidarity 
groups in each of the remaining districts. No organization surveyed reported training service providers and law enforcement in Zimba. In addition to Pemba and Siavonga, there were three districts, namely, Gwembe, Kazungula and Zimba, in which no organization surveyed reported providing financial and in-kind sustenance support (Figure 2 ). The data above mask geographic variations in the provision of interventions to mitigate barriers to prevention and negative social outcomes of HIV infection

It is also worth noting that the study found some rural-urban variations in the implementation of interventions to mitigate barriers to prevention and negative social outcomes of HIV infection. For example, training of service providers and law enforcement agencies, counseling, and legal/policy/institutional reform interventions were implemented by at least one organization surveyed in rural areas of Gwembe and Kazungula, but not in urban areas of those districts. In addition, medical and legal assistance services were not provided by organizations surveyed in urban areas of Gwembe and Sinazongwe and in rural areas of Choma and Namwala. Some rural-urban disparities are also seen in the percentage of organizations surveyed that were implementing a given intervention, the most noteworthy being training of service providers and law enforcement in Choma and counseling in Livingstone. For example, in Livingstone, eight percent of organizations surveyed implemented counseling interventions in rural compared to 13 percent in urban areas of the district. While there was a clear urban advantage in Livingstone, Chomba reported above 10 percent both in urban and rural areas.

Over all, none of the interventions to mitigate biological outcomes of HIV infection was implemented in Kazungula and Pemba by organizations surveyed while in Gwembe, Kalomo and Livingstone, four of the five interventions shown were implemented by at least one organization, but the districts differed in terms of which intervention was not provided. For example, in Gwembe, none of the organizations surveyed implemented interventions to prevent, diagnose and treat viral hepatitis. In Kalomo, it was HIV-related opportunistic infection prophylaxis and treatment that were not provided by any of the organizations surveyed. Further, none of the organizations surveyed reported implementing tuberculosis prevention, diagnosis and treatment services in Livingstone while in Monze, Sinazongwe and Zimba, only one of the interventions - palliative care - was reported to be implemented.

\section{Gender Integration}

One in every five organizations did not integrate gender issues into their HIV-prevention activities and about 46 percent integrated "most or all" gender issues. Over 70 percent of organizations survey addressed gender norms and violence against women in their HIV-prevention programming. Gender-related barriers in access to and utilization of HIV and AIDS services were addressed by 68 percent of organizations. The topics that were least likely to be addressed were gender-differentials in access to education and economic security (39 percent) and cross-generational sex (37 percent), which has been identified as one of the key drivers of HIV transmission in Zambia.

A review of the various activities conducted by program implementers to address gender issues in HIV-prevention projects in Southern Province suggests that there is a continuum of approaches. Approaches range from those addressing gender inequalities and discrimination, gender norms and gender-based violence to those that recognized women's lack of income, a general denial or lack of awareness of women's rights, and the specific vulnerabilities of adolescents and youth. Some organizations built the capacity of their own staff in gender mainstreaming and gender norms. Others focused on empowering women by improving girls' access to information about their rights in Choma, Kazungula, and Livingstone. In Choma, gender-integration activities also included disseminating information about the gender-based violence (GBV) Act of 2011[10]. In one district, gender integration also included promoting male involvement in reproductive health. Many of the activities described could be classified as social and behavioral change communication delivered through both community-based informants and networks as in Gwembe and media channels such as radio as in Livingstone and Mazabuka.

As illustrated in Figure 3, the most frequently reported activity was community education with 8 respondents, followed by education and sensitization on gender-based violence as well as girl education on human rights and reproductive health with 6 respondents each.

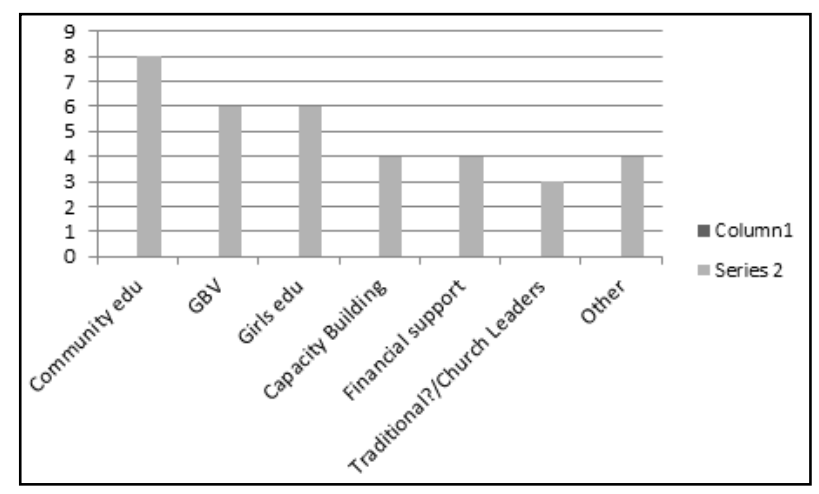

Figure 3. Gender related activities and frequency of integration in HIV prevention programs in Southern Province.

\section{Discussion}

The results of the survey reveal gaps across all the three 
focus areas: character and capacities of the NGOs involved in HIV prevention, scale and scope of the interventions and gender integration. In terms of the type and character of the NGOs, the results expose the reality that the NGOs are not evenly spread across the 12 districts of the province. A major notable gap is their concentration along the line of rail or urban-based towns of Livingstone, Mazabuka, Monze and Choma. Subsequently the rural districts suffer from lack of services, especially given the weak capacities of the government structures and the nation-wide dependency on NGOs to deliver HIV prevention services to the neediest in the country. In terms of capacity, most of the NGOs are small-scale, understaffed and under-financed in terms of over-dependency on single source of funding and absence of government funding. The implications are that the majority are not running sustainably and are short in staff competencies and capacities to deliver the needed HIV prevention programs and services. Lack of diversified funding also limits the abilities of the NGOs to diversify their activities and services across all corners of their respective geographical remits, across the province and to cater for more complex issues, especially among the key populations.

With regard to the interventions carried out by the NGOs, the results of the pilot test revealed apart geographic gaps discussed above, there is evidence of content or services gaps in the NGO HIV-prevention response. For example, no organization surveyed implemented condom social marketing, comprehensive sex/HIV education and social mobilization activities in Pemba or VCT in urban areas of Gwembe and Kazungula districts. No organization surveyed implemented mass media campaigns or interpersonal education and persuasion programs in Pemba, Siavonga, and Zimba districts. No organization implemented any interventions affecting knowledge, attitudes, and behavior in the past 12 months in urban areas of Kazungula, and to a lesser extent, in urban areas of Gwembe. While condom distribution was the most common harm reduction intervention, no organization surveyed distributed condoms in Zimba and in urban areas of Kazungula and Pemba.

Furthermore, no organization surveyed promoted or implemented voluntary medical male circumcision in 8 out of 12 districts of Southern Province, even though there is compelling evidence that male circumcision reduces the risk of heterosexually acquired HIV infection in men by approximately $60 \%$. The finding that voluntary male circumcision is generally not implemented by most organizations in Southern Province is consistent with the fact that in Southern Province, circumcision is not practiced compared to other parts of Zambia such as Northwestern and Eastern province. The gaps in condom use distribution and comprehensive sex education interventions are also in line with the prevailing pro-large family norms prevailing in the province. These interventions may be seen as working against the existing norms.

Regarding gender integration, the results also suggest both structural and content gaps. For starters, only about half of the organizations surveyed addressed gender issues in their programing. Gaps in terms of geographic spread are also apparent as some districts had lesser NGOs that mainstreamed gender issues. Also, only slightly over half addressed gender related barriers to accessing services and some crucial topics in gender and HIV prevention were hardly addressed. Unfortunately, among the crucial issues not addressed include those that are classified as key drivers of HIV spread, such as empowerment of girls, cross-generation sex, sex education and economic and educational security.

From a methodological standpoint, the pilot test revealed the need to examine dimensions of program content, quality and intensity. The questionnaires simply asked implementers whether or not a specific service was in place in a particular district and in rural or urban locations and no quantifiable data were available about the quality of HIV-prevention services or the intensity with which interventions were being delivered by the NGO sector and over which periods of time. The reference period used was 12 months. The data also do not permit the assessment of the allocative efficiency of HIV-prevention program. The questionnaires were also quite lengthy and cannot be effectively self-administered. It will be important for future adaptations of the tool kit to use an electronic module format where appropriate and to assess validity and reliability of responses provided through site visits, a document review, interviews with beneficiaries, and other means.

Despite these limitations, the study permits us to know which interventions are being implemented by the NGO sector, contributing to new knowledge in Zambia about geographic gaps in the availability of HIV-prevention interventions in the NGO sector. Districts often do not have other data sources to use for operational plans and to decide which interventions and activities should be prioritized. Compared to a routine program information system, the KYR takes a longer time to implement, and is costlier. However, the existing systems routine and non-routine information systems in Zambia cannot provide information in enough detail to show gaps in HIV-prevention response in the NGO sector, and while the Zambia has an online system for capturing which program implementing organizations are implementing broad categories of HIV-prevention interventions, the system is unable to host the detailed type of data collected by KYR. It is recognized that the KYR data cannot be collected routinely. However, a national-level KYR study can provide some sense of geographic gaps in HIV-prevention between \& within provinces. It is recommended that a KYR study be implemented every 2-3 years between DHS surveys. 


\section{Conclusions}

The fact that Southern Province in particular and Zambia as a whole reported an HIV prevalence rate averaging 13 percent among adults in 2014 is an indication that NGOs in this country still have a critical role to play in the prevention of this scourge. It is, however, important to note that this Pilot study has identified several gaps in terms of geographical areas coverage and types of interventions that are provided by the NGOs among different districts and between rural and urban parts of each district. The finding of this study may provide the basis on which the NGOs involved in providing HIV prevention responses in the Southern Province will be encouraged to network and develop strategies that will enable them to harmonise their interventions. This will not only help to reduce the chances of duplication of interventions being provided in some districts but will also make it easier for the service providers to take specific interventions to areas and populations in critical need.

\section{Acknowledgments}

The study is a product of collaboration among three organizations notably: National HIV and AIDS Council (NAC) of Zambia; Tulane University, School of Public Health and the Institute of Economic and Social Research (INESOR), of the University of Zambia. The study team thanks NAC for their leadership and support in facilitating the implementation of the study, upon which this article is based. We also express our gratitude to the Office the Permanent Secretary, Southern Province, for the valuable role in disseminating and validating the study results. Last, but not least, we acknowledge the mutually constructive and beneficial partnership between Tulane University and INESOR and specifically for the respective technical inputs.

\section{REFERENCES}

[1] Government of the Republic of Zambia. National AIDS Strategic Framework 2014 - 2016: A Nation Free from the Threat of HIV and AIDS. National HIV/AIDS/STI/TB Council, Lusaka; 2014.

[2] Central Statistical Office Zambia, Ministry of Health (MOH) [Zambia], and ICF International. Zambia Demographic and Health Survey 2013-14; 2014.

[3] Amirkhanian, Y.A, et al. HIV Prevention Non-Governmental Organizations in Central and Eastern Europe: Programs, Resources and Challenges; 2004.

[4] Fernandez, M.I, et al. HIV prevention programs of nongovernmental organizations in Latin America and the Caribbean: The Global AIDS Intervention Network project; 2005.

[5] Rau, Bill. The Politics of Civil Society in Confronting HIV/AIDS.

http://www.jstor.org/stable/pdf/3569421.pdf?refreqid=exce 1sior:26fa618e988fad4cc278c66ce5379f40. Accessed, 28/05/2-17.

[6] Ndimbwa T, Emanuel M and Mushi E. The Role Played by NGOs in Preventing the Spread of HIV/ AIDS and Supporting People Living with HIV/AIDS in Tanzania: A Case of Dar es Salaam Region. International Journal of Academic Research in Business and Social Sciences, Vol. 3, No. 11, Nov. 2013

[7] Jewkes R, et al. Impact of Stepping Stones on incidence of HIV and HSV-2 and sexual behaviour in rural South Africa: cluster randomised controlled trial. BMJ 2008; 337: a506. http://www.bmj.com/content/bmj/337/bmj.a506.full.pdf. Accessed 28.05.2017.

[8] Hughes-d'Aeth A. Evaluation of HIV/AIDS peer education projects in Zambia. Evaluation and Program Planning 25 (2002) 397-407. 2002.

[9] Sweat, Michael. A Framework for Classifying HIV Prevention Interventions. Geneva, Switzerland, Joint United Nations Programme on HIV/AIDS (UNAIDS), 2008.

[10] Government of the Republic of Zambia. Anti-Gender Based Violence Act 2011.

[11] Sweat, Michael.2008 Report to the Joint United Nations Programme on HIV/AIDS (UNAIDS). A Framework for Classifying HIV Prevention Interventions. 